\title{
Ferroptosis due to Cystathionine $\gamma$ Lyase/Hydrogen Sulfide Downregulation Under High Hydrostatic Pressure Exacerbates VSMC Dysfunction
}

\author{
Ruxi Jin, Ruixue Yang, Changting Cui, Haizeng Zhang, Jun Cai, Bin Geng * and \\ Zhenzhen Chen*
}

State Key Laboratory of Cardiovascular Disease, National Center for Cardiovascular Diseases, Hypertension Center, Fuwai Hospital, Chinese Academy of Medical Sciences and Peking Union Medical College, Beijing, China

OPEN ACCESS

Edited by:

Zhi Qi,

Nankai University, China

Reviewed by:

Yongsheng Chang,

Tianjin Medical University, China

Jinwei Tian,

The Second Affiliated Hospital of Harbin Medical University, China

${ }^{*}$ Correspondence:

Bin Geng

bingeng@hsc.pku.edu.cn

Zhenzhen Chen

chenzhenzhen@bjmu.edu.cn

Specialty section:

This article was submitted to

Cell Death and Survival,

a section of the journal

Frontiers in Cell and Developmental

Biology

Received: 05 December 2021

Accepted: 10 January 2022

Published: 03 February 2022

Citation:

Jin $R$, Yang $R$, Cui C, Zhang $H$, Cai J, Geng $B$ and Chen $Z$ (2022) Ferroptosis

due to Cystathionine $\gamma$ Lyase/ Hydrogen Sulfide Downregulation Under High Hydrostatic Pressure Exacerbates VSMC Dysfunction. Front. Cell Dev. Biol. 10:829316. doi: 10.3389/fcell.2022.829316
Hydrostatic pressure, stretch, and shear are major biomechanical forces of vessels and play critical roles in genesis and development of hypertension. Our previous work demonstrated that high hydrostatic pressure (HHP) promoted vascular smooth muscle cells (VSMCs) two novel subsets: inflammatory and endothelial function inhibitory VSMCs and then exacerbated VSMC dysfunction. However, the underlying mechanism remains unknown. Here, we first identified that aortic GPX4 (a core regulator of ferroptosis) significantly downregulated association with VSMC novel phenotype elevation in SHR rats and hypertension patients. In primary VSMCs, HHP $(200 \mathrm{mmHg})$ increased iron accumulation, ROS production, and lipid peroxidation compared with normal pressure (100 mmHg). Consistently, the ferroptosis-related gene (COX-2, TFRC, ACSL4, and NOX1) expression was also upregulated. The ferroptosis inhibitor ferrostatin-1 (Fer-1) administration blocked HHP-induced VSMC inflammatory (CXCL2 expression) and endothelial function inhibitory (AKR1C2 expression) phenotyping switch association with elevation in the GPX4 expression, reduction in the reactive oxygen species (ROS), and lipid peroxidation production. In contrast, the ferroptosis inducer RLS3 increased HHP-induced CXCL2 and AKR1C2 expressions. These data indicate HHP-triggering ferroptosis contributes to VSMC inflammatory and endothelial function inhibitory phenotyping switch. In mechanism, HHP reduced the VSMC GSH content and cystathionine gamma-lyase (CSE)/hydrogen sulfide $\left(\mathrm{H}_{2} \mathrm{~S}\right)$-an essential system for GSH generation. Supplementation of the $\mathrm{H}_{2} \mathrm{~S}$ donor-NaHS increased the VSMC GSH level, alleviated iron deposit, ROS and lipid peroxidation production. NaHS administration rescues both HHP- and RLS3-induced ferroptosis. Collectively, HHP downregulated VSMC CSE $/ \mathrm{H}_{2} \mathrm{~S}$ triggering GSH level reduction, resulting in ferroptosis, which contributed to the genesis of VSMC inflammation and endothelial function inhibitory phenotypes.

Keywords: hydrostatic pressure, ferroptosis, hydrogen sulfide, VSMCs, hypertension 


\section{INTRODUCTION}

Biomechanical forces within the vasculature contain wall shear stress, circumferential wall tensile stress, and hydrostatic pressure (Takayama et al., 2013), and these biomechanics contribute to pathogenesis and development of hypertension and its complications (Hayashi and Naiki, 2009). Shear stress disorder impaired endothelial function and involved in vascular remodeling, atherosclerosis, plaque progression, and vascular malformations (Zhou et al., 2014). The mechanical cyclic stretch force also contributes to vascular smooth muscle cell functions (e.g., apoptosis, proliferation, and migration), which is crucial in vascular remodeling during hypertension (Cheng et al., 2012; Qi et al., 2018), whereas little attention is paid to hydrostatic pressure regulation. A recent study has shown that high hydrostatic pressure (HHP)-driving VSMCs differentiated into inflammatory subset (elevating CXCL2, CXCL3, and CCL2) and endothelial function inhibitory subset (upregulating AKR1C2, AKR1C3, and PEDF) by single-cell sequencing and exacerbated the VSMC dysfunction (Chen Z. et al., 2021). However, the underlying mechanism remains unknown.

Ferroptosis is a novel type of programmed cell death, characteristic as iron overload, reactive oxygen species (ROS) accumulation, iron-dependent lipid peroxidation, and mitochondrial shrinkage ( $\mathrm{Wu}$ et al., 2021). Ferroptosis is triggered by the cystine/glutamate antiporter system $\left(\mathrm{Xc}^{-}\right.$ system) dysfunction, glutathione (GSH) depletion, and glutathione-dependent antioxidant enzyme glutathione peroxidase 4 (GPX4) inactivation (Yang and Stockwell, 2016). GSH is an important antioxidant, synthesized from cysteine and oxidizes to oxidized glutathione (GSSG) dependent on GPX4 (Badgley et al., 2020). Meanwhile, GPX4 also reduced cytotoxic lipid peroxide $(\mathrm{L}-\mathrm{OOH})$ into nontoxic alcohol $\mathrm{L}-\mathrm{OH}$ in the presence of the cofactor GSH (Stockwell et al., 2017). Thus, GPX4/GSH is an essential regulatory system in ferroptosis. Recently, many studies highlighted that ferroptosis is a crucial pathophysiological process in chronic diseases such as cancer, diabetes, and nervous system disease (Qiu et al., 2020). Over iron deposits induced ferroptosis causing cardiomyopathy (Gujja et al., 2010) or development of vulnerable plaques (Vinchi et al., 2020). Recombinant human GPX4 (Liu et al., 2021) and ferroptosis inhibitor Fer-1 (Li et al., 2020) treatment attenuated myocardial injuries. These works indicate that ferroptosis contributes to the pathogenesis of cardiovascular diseases.

Cystathionine- $\gamma$-lyase (CSE) endogenously produces hydrogen sulfide $\left(\mathrm{H}_{2} \mathrm{~S}\right)$ exerting a cardiovascular protective role and as the key enzyme for L-cysteine (precursor of GSH) (Kimura, 2021). $\mathrm{H}_{2} \mathrm{~S}$ plays an antioxidative role by facilitating the GSH content and removing ROS (Tabassum and Jeong, 2019), also inhibiting GPX4 activity and maintaining the $\mathrm{Xc}^{-}$ system stability to mitigate ferroptosis (Chen S. et al., 2021; Wang et al., 2021b). In the present study, we seek to clarify whether VSMC ferroptosis involve into the HHP-induced VSMC phenotyping switch. In mechanism, we investigate the $\mathrm{CSE} / \mathrm{H}_{2} \mathrm{~S}$-dependent GSH level in ferroptosis in the HHP condition.

\section{MATERIALS AND METHODS}

\section{Human Samples}

Human internal mammary arteries were obtained from patients undergoing off-pump coronary artery bypass graft. The internal mammary artery (about $5 \mathrm{~mm}$ ) was acquired from the patient's surgical donor artery; then, the paraffin slices were prepared for immunofluorescent staining. This study was approved by Fuwai Hospital Ethics Committee and performed in accordance with ethical standards.

\section{Animal and Materials}

Adult male Wistar-Kyoto (WKY) and spontaneously hypertensive rats (SHR) aged 12-16 weeks were housed in controlled temperature at a 12:12-h light-dark cycle with free access to water and standard diet. All animal protocols complied with all relevant ethical regulations and were approved by the Institutional Animal Care and Use Committee, the Experimental Animal Center, Fuwai Hospital, and the National Center for Cardiovascular Diseases, China. The primary antibodies used in this study are as follows: anti-GPX4 (ab125066, Abcam); antiCXCL2 (PA5-47015, Invitrogen); anti-AKR1C2 (ab166900, Abcam); anti-CSE (ab151769); ferrostatin-1 (S7243, Selleck); RLS3 (S8155, Selleck); and NaHS (161527, Sigma).

\section{$\mathrm{H}_{2} \mathrm{~S}$ Production Measurement}

$\mathrm{H}_{2} \mathrm{~S}$ production was measured by the modified methylene blue method, as mentioned previously (Niu et al., 2021). In detail, the inner ring of the Erlenmeyer flask was added with zinc acetate and a filter paper to absorb $\mathrm{H}_{2} \mathrm{~S}$. The outer ring was added with an incubation buffer (potassium phosphate buffer, L-cysteine and pyridoxal $5^{\prime}$-phosphate). The cell lysate in the potassium phosphate buffer was added into the outer ring of the conical flasks. The reaction was performed in $37^{\circ} \mathrm{C}$ shaking water bath by incubation. After sufficient reaction, trichloroacetic acid was added into the outer ring and incubated for another $1 \mathrm{~h}$ to terminate the reaction. Followed by adding N, N-dimethylp-phenylenediamine sulfate and $10 \%$ ammonium ferric sulfate, absorbance at $670 \mathrm{~nm}$ was measured by spectrophotometry.

\section{Cell Culture and Treatment}

Primary human aorta vascular smooth muscle cells (HASMCs) were purchased from ScienCell Research Laboratories, Inc. HAMSCs were cultured in a smooth muscle cell medium (1,101, ScienCell Research Laboratories.) containing 2\% FBS, $100 \mathrm{U} / \mathrm{mL}$ penicillin-streptomycin, and $1 \%$ smooth muscle cell growth factor at $37^{\circ} \mathrm{C}$ in a $5 \% \mathrm{CO}_{2}$ atmosphere. HASMCs in passages three to six were used in this study. We used a specific hydrostatic pressure device for hydrostatic pressure treatment, as described previously (Chen Z. et al., 2021). HASMCs ( 70\% confluence) were set in a hydrostatic pressure device at $100 \mathrm{mmHg}$ or $200 \mathrm{mmHg}$ for culturing for $24 \mathrm{~h}$. For inhibition or activation of ferroptosis, HASMCs were treated with Fer-1 $(10 \mu \mathrm{M}, 24 \mathrm{~h})$ or RLS3 $(0.1 \mu \mathrm{M}, 6 \mathrm{~h})$ under hydrostatic pressure. For increasing exogenous $\mathrm{H}_{2} \mathrm{~S}$, HASMCs were treated with NaHS $(100 \mu \mathrm{M}, 24 \mathrm{~h})$ under hydrostatic pressure. 


\section{Primary Mouse Vascular Smooth Muscle Cell Isolation}

Aortic smooth muscle cells were isolated from wild-type male 6-8-week and CSE-knockout mice. Mice were sacrificed by decapitation, followed by separating the aorta via removing the fatty tissue and vascular adventitia. Then, the mouse aorta was cut into pieces and allowed for digestion for $8 \mathrm{~h}$ in the DMEM containing collagenase type 1 . The obtained VSMCs were centrifuged at $300 \mathrm{xg}$ for $5 \mathrm{~min}$, and the pellet was resuspended in the DMEM supplemented with $10 \%$ FBS, $2 \mathrm{mM}$ L-glutamine, and $100 \mathrm{U} / \mathrm{mL}$ penicillin/streptomycin. The medium was replaced every 2 days. All experimental procedures were conducted in an incubator at a temperature of $37^{\circ} \mathrm{C}$, in an atmosphere of $95 \%$ air and $5 \% \mathrm{CO}_{2}$. Primary SMCs with the passage number three to eight were used in the study.

\section{Iron and GSH Detection}

We used two different methods to detect the iron content. After treatment, the intracellular ferrous iron level was assessed with an iron colorimetric assay kit (ab83366, Abcam) according to the manufacturer's instruction protocol. For FeRhoNox-1 staining, $5 \mu \mathrm{M}$ of the FeRhoNox-1 staining working solution (MX4558 and $\mathrm{MKBIO}$ ) was added and incubated in a $37^{\circ} \mathrm{C}, 5 \% \mathrm{CO}_{2}$ incubator for $60 \mathrm{~min}$ after treatment for $24 \mathrm{~h}$ in a hydrostatic pressure chamber. After washing three times with PBS, cells were imaged with a confocal microscope. The cellular level of glutathione was determined using the Glutathione Assay Kit (S0035, Beyotime) according to the manufacturer's instruction protocol.

\section{Reactive Oxygen Species (ROS) Measurement}

ROS was measured by using a fluorometric intracellular ROS kit (MAK143, sigma). HASMCs were incubated with $10 \mu \mathrm{M}$ DCFHDA for $1 \mathrm{~h}$. After washing with PBS three times, fluorescence signals were observed by using a fluorescence microscope.

\section{Mitochondrial Superoxide Measurement}

We use the MitoSOX ${ }^{\mathrm{TM}}$ Red mitochondrial superoxide indicator (M36008, Invitrogen) to detect ROS in the mitochondria. After treatment, HASMCs were stained with $5 \mu \mathrm{M}$ MitoSOX Red for $10 \mathrm{~min}$, followed by washing with PBS. The images were observed by using a confocal microscope.

\section{Lipid Peroxidation Detection}

HASMCs were labeled with a lipid peroxidation sensor BODIPY ${ }^{\mathrm{TM}}$ $581 / 591 \mathrm{C} 11$ (D3861, Invitrogen) at $2 \mu \mathrm{M}$ in a living cell imaging solution for $1 \mathrm{~h}$ at $37^{\circ} \mathrm{C}$. The images were observed by using a confocal microscope. The fluorescence signals correspond to the oxidized (orange) form of BODIPY 581/591 C11. Oxidation of BODIPY-C11 was calculated by the orange fluorescence intensity.

\section{Immunofluorescence Staining}

For aorta immunofluorescent staining, paraffin sections were incubated with $3 \%$ hydrogen peroxide to block the endogenous peroxidase activity. Antigen retrieval was performed for $20 \mathrm{~min}$ at $97^{\circ} \mathrm{C}$ in a citrate buffer, and sections were cooled to room temperature. For HAMSC immunofluorescent staining, cells were fixed with $4 \%$ paraformaldehyde for $15 \mathrm{~min}$ at room temperature. After washed with PBS three times, cells were permeabilized with $0.5 \%$ Triton $\mathrm{X}-100$ for $15 \mathrm{~min}$ at room temperature. Subsequently, above tissue sections or cells were blocked with $1 \%$ BSA for $1 \mathrm{~h}$ and incubated with the primary antibody at $4^{\circ} \mathrm{C}$ overnight, followed by incubating with a fluorescent-labeled secondary antibody for $1 \mathrm{~h}$ at room temperature according to the manufacturer's instructions. After washing three times with PBS, the nuclei were stained with Hoechst for $8 \mathrm{~min}$. The images of tissues or cells were collected using a fluorescence microscope or by confocal microscopy.

\section{Western Blot}

Cells were lysed in RIPA buffer containing protease- and phosphatase-inhibitor cocktails, followed by sonication and centrifugation. Total protein of cells was quantified by BCA assay. For the assay, $40 \mu \mathrm{g}$ protein samples were separated by SDS-PAGE and transferred to polyvinylidene fluoride membranes. Then, the PVDF membranes were blocked by $5 \%$ defatted milk for $1 \mathrm{~h}$ and probed with the primary antibody at $4^{\circ} \mathrm{C}$ overnight. After washing and incubating with the horseradish peroxidase-conjugated secondary antibody for $1 \mathrm{~h}$ at room temperature, the membranes were visualized using the chemiluminescence kit.

\section{RNA Extraction and qRT-PCR}

Total RNA from HASMCs was extracted using TRIzol reagent (Invitrogen) according to the manufacturer's instructions. cDNA was reverse transcribed from $1 \mu \mathrm{g}$ total RNA by using the cDNA synthesis kit (K1622, Thermo scientific). The real-time PCR was performed in a final volume of $20 \mu \mathrm{l}$, which contained $10 \mu \mathrm{l}$ of the $2 \times$ SYBR Mix (Yeasen, China), $1 \mu \mathrm{l}$ of forward and reverse primers, respectively, $4 \mu \mathrm{l}$ of template cDNA, and $5 \mu \mathrm{l}$ of RNase-free $\mathrm{H}_{2} \mathrm{O}$. A sample without cDNA was subjected to an identical protocol as a negative control. The PCR amplification was accomplished with initial denaturation at $95^{\circ} \mathrm{C}$ for $10 \mathrm{~min}$, followed by 40 cycles at $95^{\circ} \mathrm{C}$ for $15 \mathrm{~s}$ and $1 \mathrm{~min}$ at $60^{\circ} \mathrm{C}$ for primer annealing and extension. The relative expression of target genes was normalized to that of GAPDH and analyzed by the $2^{-\Delta \Delta \mathrm{CT}}$ method. The primer sequences used for qRT-PCR are provided in Supplementary Table S1.

\section{Statistical Analysis}

All data are presented as the mean \pm SD. The statistical significance of differences between groups was analyzed by the $t$-test or by one-way analysis of variance (ANOVA) when more than two groups were compared. $p$ values $<0.05$ were considered as statistically significant.

\section{RESULTS}

\section{Elevation of Ferroptosis in Aortic Media of SHR and Hypertensive Patients Associated With VSMC Novel Phenotypes}

High hydrostatic pressure drives VSMC differentiation into two novel phenotypes: the inflammatory phenotype (marker genes 

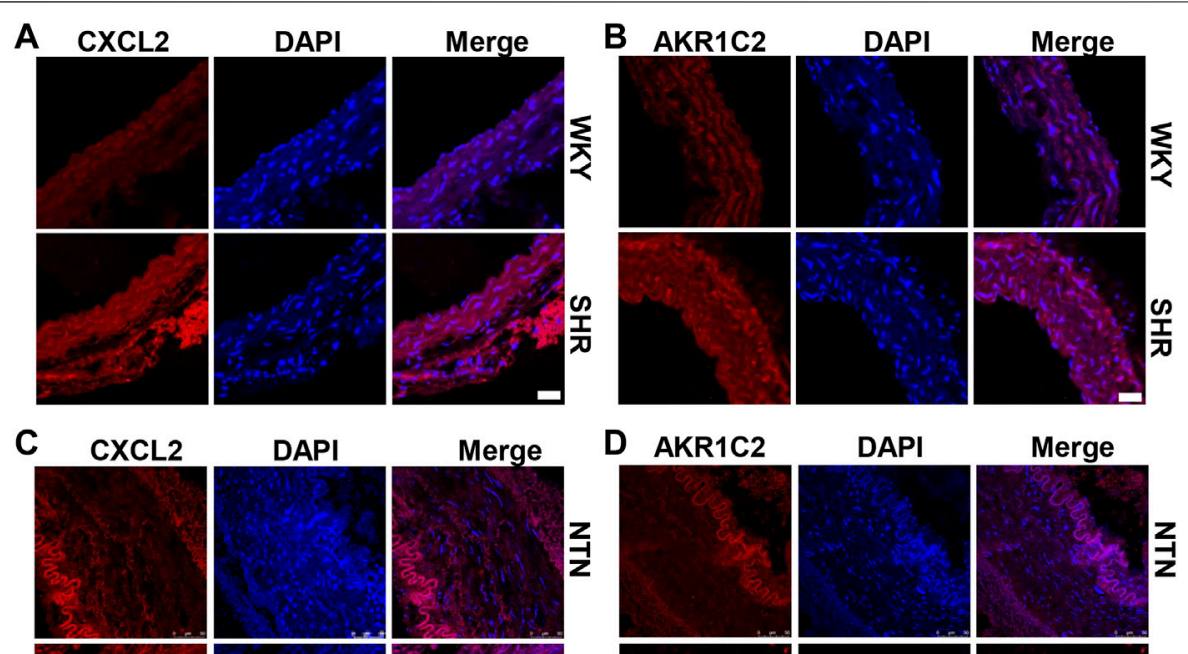

Merge
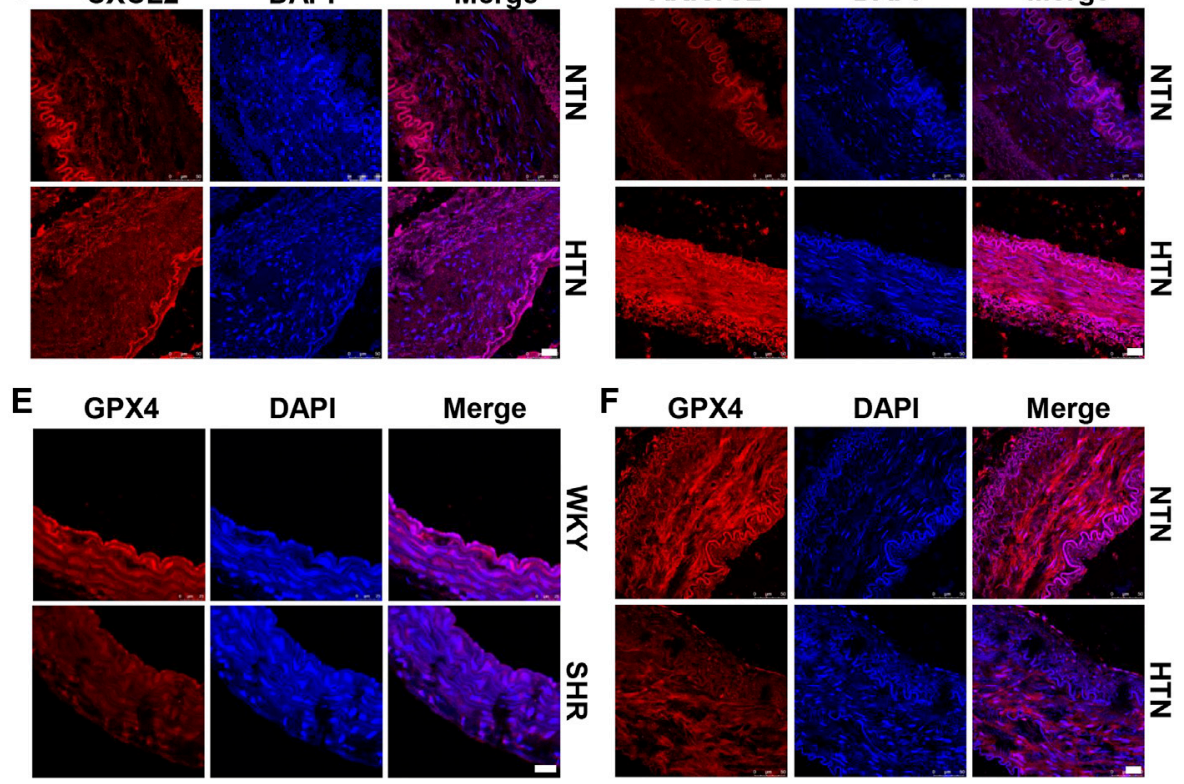

FIGURE 1 | GPX4 expression positively correlated with the VSMC-specific phenotype in SHR and hypertensive patients. Immunofluorescent staining of CXCL2 (A) and AKR1C2 (B) in the arterial media of SHR. Immunofluorescence of CXCL2 (C) and AKR1C2 (D) on paraffin-embedded human normotension (NTN) and hypertension (HTN) internal mammary arteries. The GPX4 expression was detected by immunofluorescent staining in SHR (E) and human hypertensive patients (F). Nuclei were counterstained with Hoechst. Scale bar $=25 \mu \mathrm{M}$.

are CXCL2/CXCL3/CCL2) and endothelial function inhibitory phenotype (marker genes are AKR1C2/AKR1C3/PEDF), and promote VSMC dysfunction (Chen Z. et al., 2021). Compared with WKY rats, SHR exhibits CXCL2 and AKR1C2 increase in the media of aorta (Figures 1A,B and Supplementary Figure S1A). Similarly, CXCL2 and AKR1C2 expressions were significantly enhanced in the human internal mammary artery of hypertensive patients in comparison with normotensive patients (Figures 1C,D and Supplementary Figure S1B). To investigate ferroptosis involved in HHPinduced VSMC novel phenotypes, we measured the GPX4 (a key enzyme of ferroptosis) protein level. Here, we showed that aortic media GPX4 dramatically downregulated in the SHR and hypertensive patients compared with the related control (Figures 1E,F and Supplementary Figure S1A-B). These results indicated that ferroptosis associated with the VSMC phenotype switch in the hypertensive animal model and hypertensive patients.

\section{High Hydrostatic Pressure Induces Ferroptosis}

To seek whether HHP induces VSMC ferroptosis, we constructed a specific cell culture chamber and set $200 \mathrm{mmHg}$ as HHP and $100 \mathrm{mmHg}$ as normal (simulates hydrostatic pressure of the aortic wall in normotensive and hypertensive individuals). For evaluation ferroptosis, HHP $(200 \mathrm{mmHg})$-treatment increased the VSMC total ROS production (Figure 2A and Supplementary Figure S2A), mitochondria-derived ROS releasing by MitoSOX Red staining (Figure 2B and Supplementary Figure S2B), lipid peroxidation production by BODIPY 588/591 C11 (Figure 2C and Supplementary Figure S2C), iron accumulation by FeRhoNox-1 staining (Figure 2D), and iron content by the iron assay kit (Figure 2E) in comparison to normal pressure. In line with the ROS enhancing and lipid peroxidation, the GPX4 protein (Figure 2F) and mRNA level (Figure 2G) also lowered under the HHP condition. Indeed, the ferroptosis-related gene (COX-2, TFRC, ACSL4, and NOX-1) mRNA expression was 


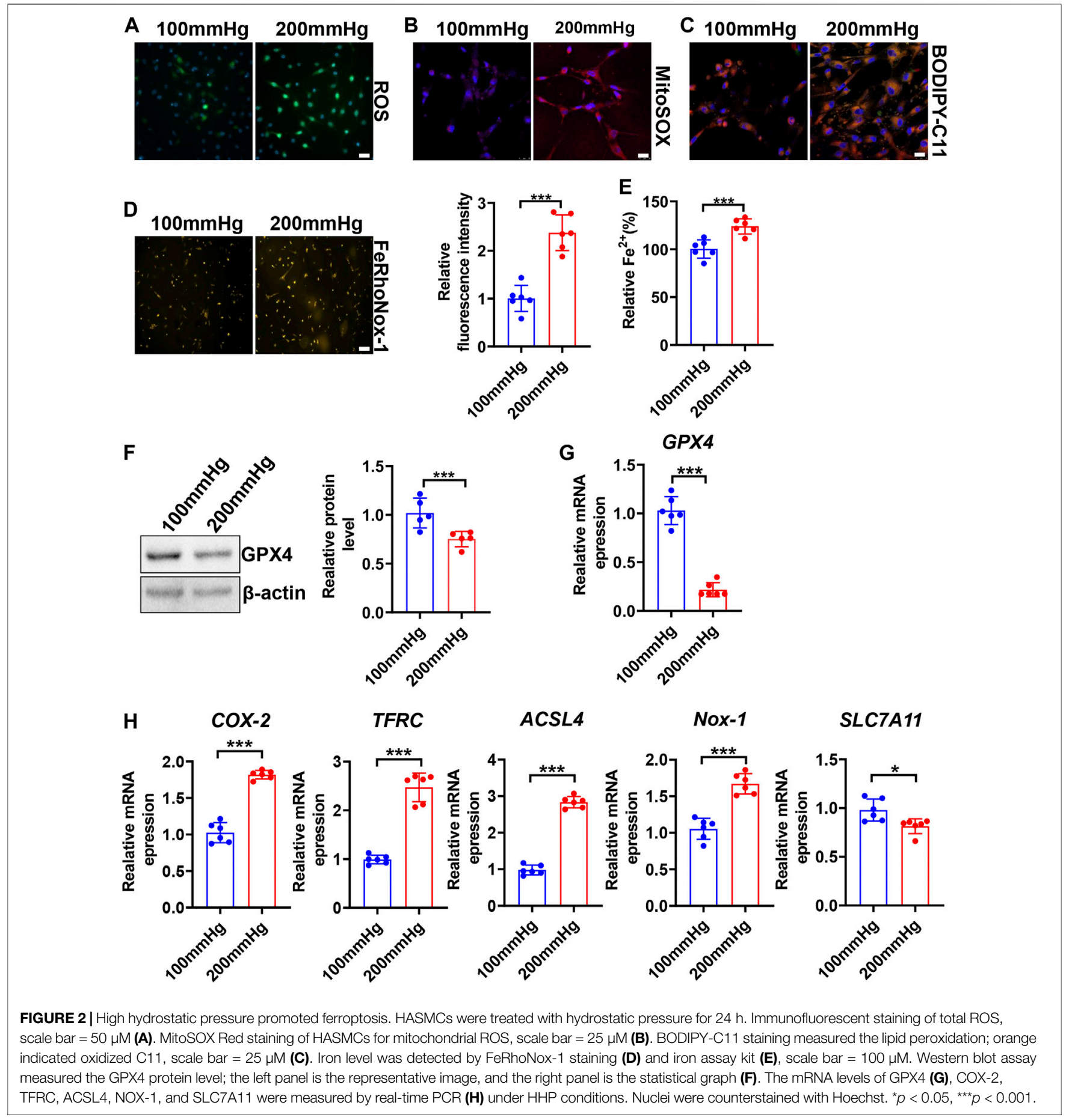

upregulated, and SLC7A11 mRNA was downregulated while exposed to HHP (Figure 2H). Overall, these results indicate high hydrostatic pressure promotes VSMC ferroptosis.

\section{Ferroptosis Contributes to the HHP-Induced Specific VSMC Phenotype Switch}

To investigate the effect of ferroptosis on HHP-induced specific VSMC subsets, we treated HASMCs with the ferroptosis suppressor Fer-1. Fer-1 supplementation upregulated the VSMC GPX4 expression under normal or high hydrostatic pressure conditions (Figure 3A). In line with GPX4 upregulation, Fer-1 administration also blocked HHPstimulated ROS production (Figure 3B), mitochondrial ROS production (Figure 3C), and lipid oxidation (Figure 3D), similar to other ferroptosis inducers (Dixon et al., 2012). These results confirmed Fer-1 blocked HHP-associated ferroptosis. In association with ferroptosis inhibition, Fer-1 

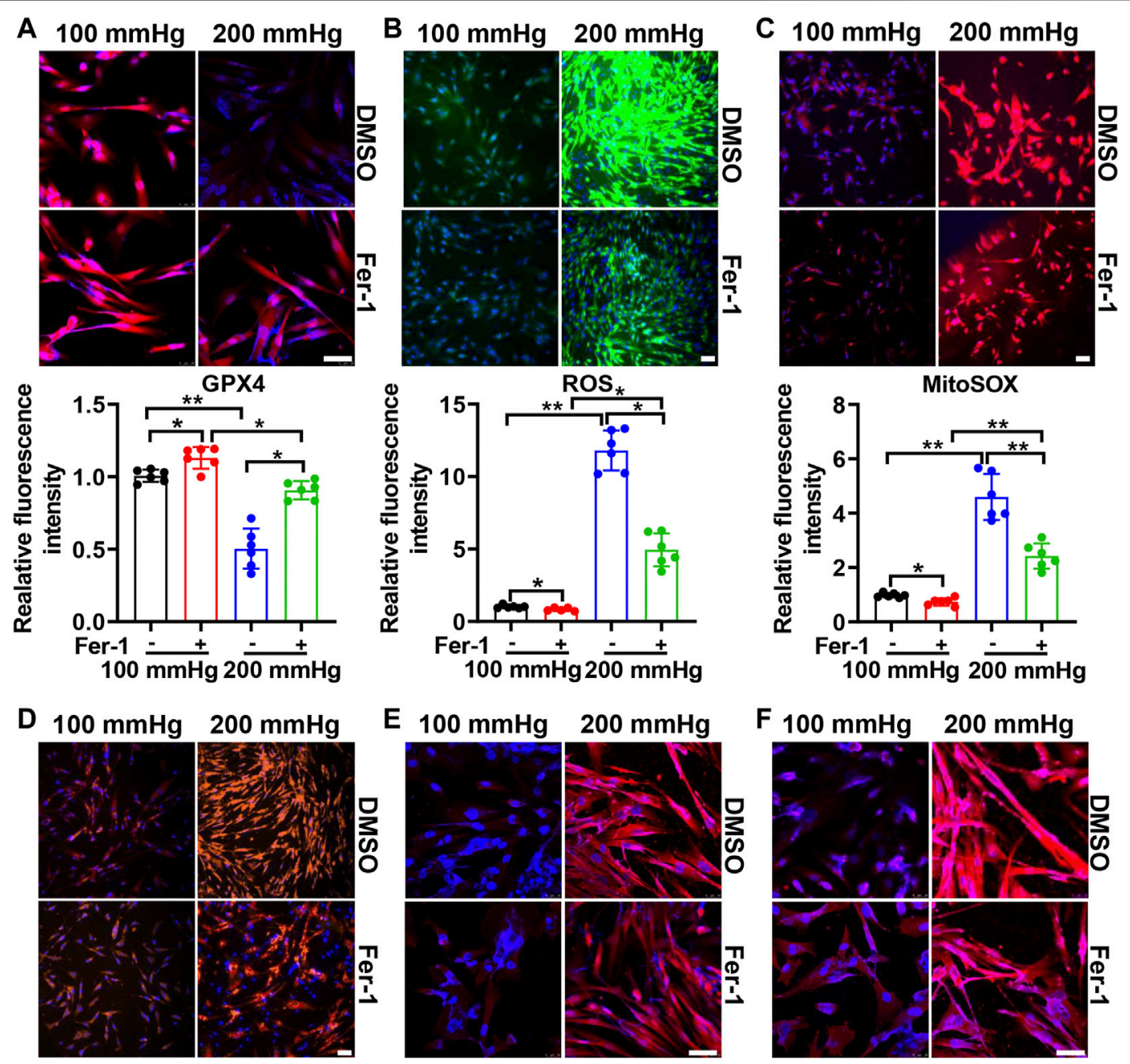

\section{E $100 \mathrm{mmHg} 200 \mathrm{mmHg}$}

\section{$\mathrm{F} 100 \mathrm{mmHg} \quad 200 \mathrm{mmHg}$}
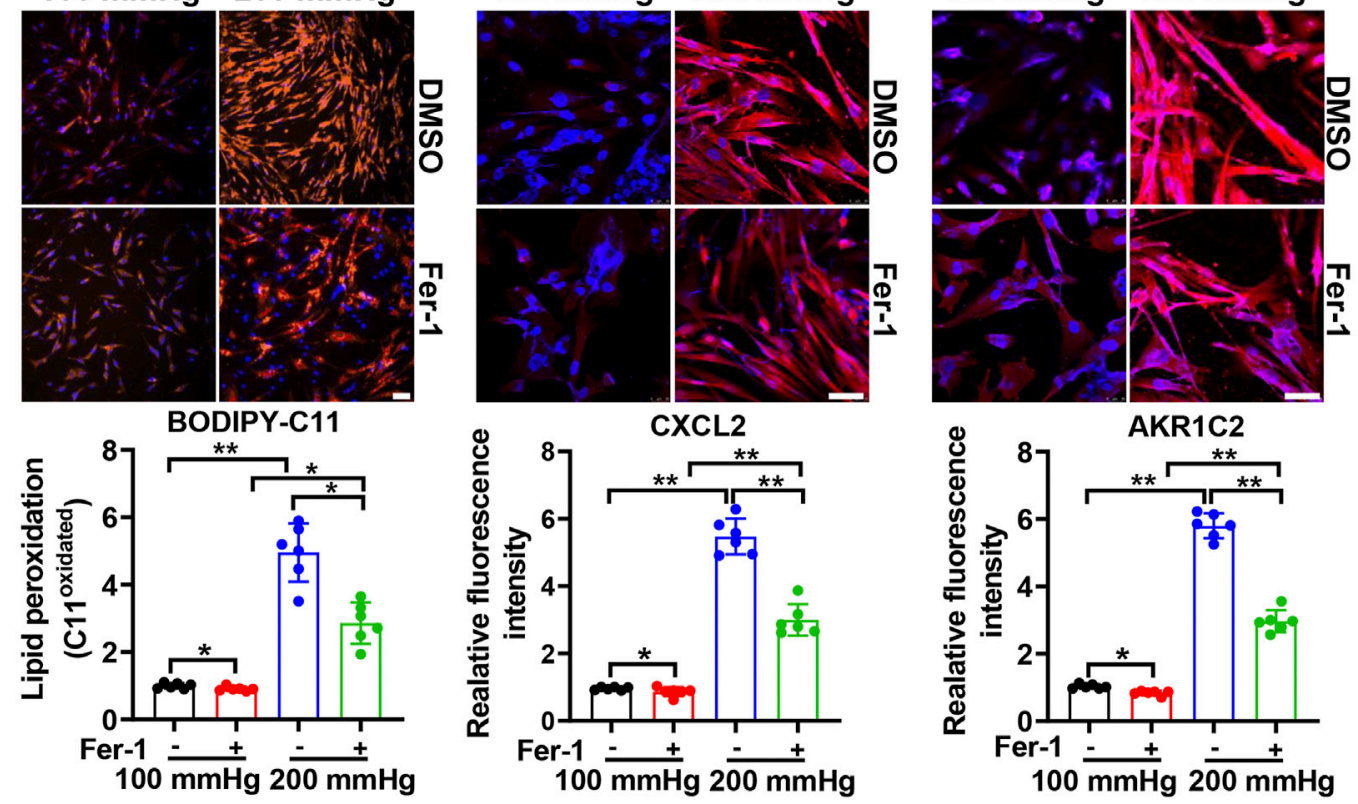

FIGURE 3 | Ferroptosis inhibitor Fer-1 mitigates the HHP-induced VSMC phenotype switch. HASMCs in a $100 \mathrm{mmHg}$ or $200 \mathrm{mmHg}$ incubator were treated with or without $10 \mu \mathrm{M}$ Fer-1 for $24 \mathrm{~h}$. The GPX4 protein level was evaluated by immunofluorescent staining; the upper panel is the representative image, and the lower panel is the statistical graph (A). ROS production was determined by the ROS staining kit; the upper panel is the representative image, and the lower panel is the statistical graph (B). Mitochondrial ROS was visualized by use of the fluorescent probe MitoSOX Red (C). BODIPY 581/591 C11 lipid oxidation in HASMCs was measured by immunofluorescent staining; the statistical graph is the oxidized (orange signal) BODIPY 581/591 C11 fluorescence intensity (D). Confocal images of the CXCL2 expression (E) and AKR1C2 expression (F) in HASMCs. Nuclei were counterstained with Hoechst. All scale bar $=50 \mu \mathrm{M}{ }^{\star} p<0.05,{ }^{\star \star} p<0.01,{ }^{\star \star \star} p<0.001$.

also blocked HHP-induced VSMC CXCL2 (Figure 3E) and AKR1C2 (Figure 3F) elevation.

Since HHP-induced ferroptosis is in part of the GPX4/GSH pathway, we used a ferroptosis inducer RLS3 (GPX4 inactivation) to confirm its role in the VSMC novel phenotype switch. Under $200 \mathrm{mmHg}$ hydrostatic pressure condition, RLS3 ulteriorly reduced the GPX4 expression (Figure 4A). Consistently, RLS3 exacerbated ferroptosis by increasing ROS production (Figure 4B), mitochondrial ROS production(Figure 4C), and lipid oxidation (Figure 4D). Correspondingly, RLS3 further increases the HHP-induced VSMC CXCL2 (Figure 4E) and AKR1C2 expression (Figure 4F). Taken together, these data 

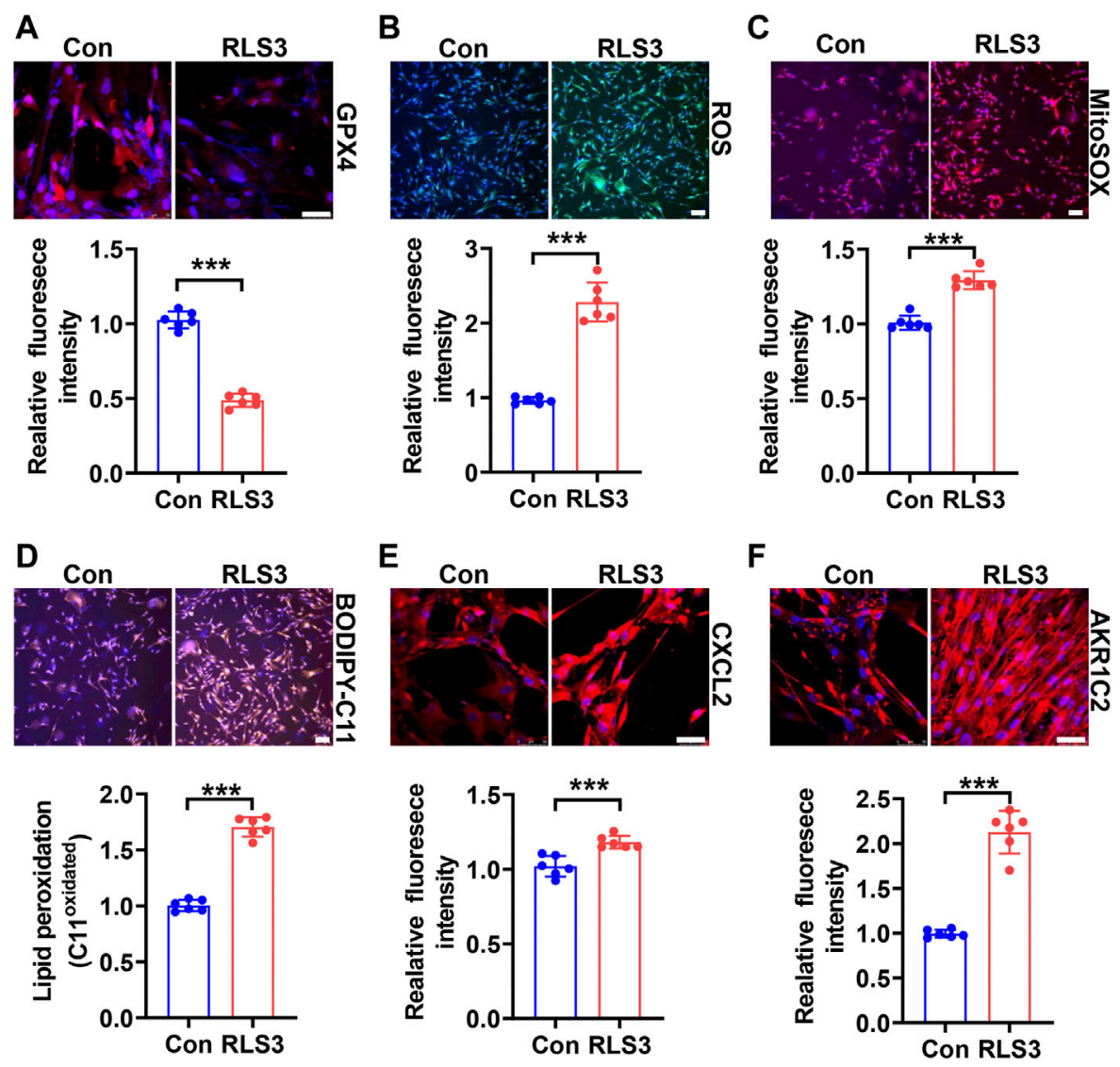

FIGURE 4 | Ferroptosis inducer RLS3 aggravates VSMC dysfunction in response to HHP. HASMCs were treated with RLS3 $0.1 \mu \mathrm{M}$ for $6 \mathrm{~h}$ in the presence of $200 \mathrm{mmHg}$. Immunofluorescent staining of GPX4 (red) in HASMCs (A). Intracellular ROS were measured by ROS staining; the upper panel is the representative image, and the lower panel is the statistical graph (B). HASMCs were labeled with the MitoSOX Red probe to detect mitochondrial ROS (C). Confocal images of the BODIPY 588/591 C11 staining; lipid oxidation was measured by the relative intensity of oxidized (orange fluorescence signal) BODIPY 581/591 C11 (D). CXCL2 (E), and AKR1C2 (F) protein levels were detected by immunofluorescent staining. All scale bar $=50 \mu \mathrm{M}{ }^{* \star *} p<0.001$.

indicated that ferroptosis contributed to the pathophysiological process of HHP-induced VSMC-specific phenotypes.

\section{$\mathrm{CSE} / \mathrm{H}_{2} \mathrm{~S}$ Alleviate HHP-Induced VSMC Ferroptosis}

$\mathrm{CSE} / \mathrm{H}_{2} \mathrm{~S}$ plays a crucial role in GSH synthesis due to controlling the GSH precursor-L-cysteine generation, and $\mathrm{CSE} / \mathrm{H}_{2} \mathrm{~S}$ plays an essential protection in VSMC function (Kimura, 2021). To investigate whether $\mathrm{CSE} / \mathrm{H}_{2} \mathrm{~S}$ involved in the modulation of HHP-induced ferroptosis, we first measured the $\mathrm{CSE} / \mathrm{H}_{2} \mathrm{~S}$ changes exposed to HHP $(200 \mathrm{mmHg})$. As Figure 5A showed, HHP treatment for $48 \mathrm{~h}$ dramatically reduced the HASMC $\mathrm{H}_{2} \mathrm{~S}$ production (about 27\%). The CSE protein (Figure 5B) and mRNA expression (Figure 5C) were also downregulated under HHP. The association with $\mathrm{CSE} / \mathrm{H}_{2} \mathrm{~S}$ downregulation and the intracellular GSH level reduced about 28\% (Figure 5D).

In contrast, supplementation $\mathrm{H}_{2} \mathrm{~S}$ donor-NaHS under HHP culture, upregulated the GSH level and GPX4 expression (Figures 5E,F and Supplementary Figure S3A) but lowered the HASMC iron concentration (Figure 5G and Supplementary Figure S3B),
ROS production (Figure 5H and Supplementary Figure S3C), mitochondrial ROS production (Figure 5I and Supplementary Figure S3D), and lipid peroxidative production (Figure 5J and Supplementary Figure S3E). Next, we isolated primary CSEknockout mouse VSMCs to confirm its role in HHP-induced ferroptosis. Under HHP conditions, CSE deficiency further decreased the GPX4 expression (Figure $\mathbf{5 K}$ and Supplementary Figure S3F), enhanced iron accumulation (Figure 5L and Supplementary Figure S3G), cellular and mitochondrial ROS (Figure $\mathbf{5} \mathbf{M}-\mathbf{N}$ and Supplementary Figure S3H-I), and lipid peroxidative production (Figure 50 and Supplementary Figure S3J). Our present data highlight HHP decreases $\mathrm{CSE} / \mathrm{H}_{2} \mathrm{~S}$ causing $\mathrm{GSH}$ level reduction attribution to pathogenesis of VSMC ferroptosis.

To confirm the effect of CSE $/ \mathrm{H}_{2} \mathrm{~S}$ on ferroptosis, we first used RLS3 to induce ferroptosis. In line with the above findings, NaHS administration also rescued the RLS3-induced GPX4 expression (Figure 6A and Supplementary Figure S4A), iron accumulation (Figure 6B and Supplementary Figure S4B), ROS production (Figure 6C and Supplementary Figure S4C), mitochondrial ROS production (Figure 6D and Supplementary Figure S4D), and 

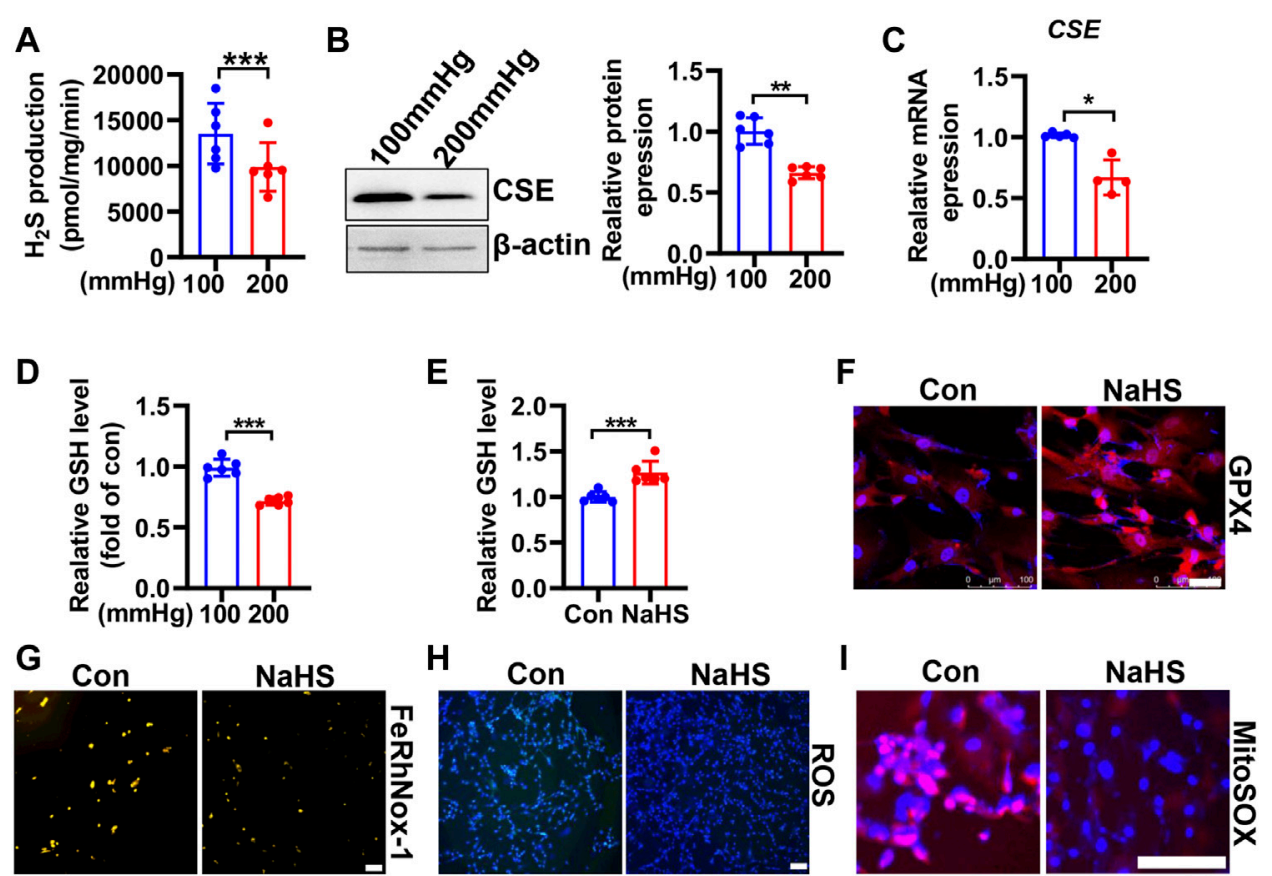

H
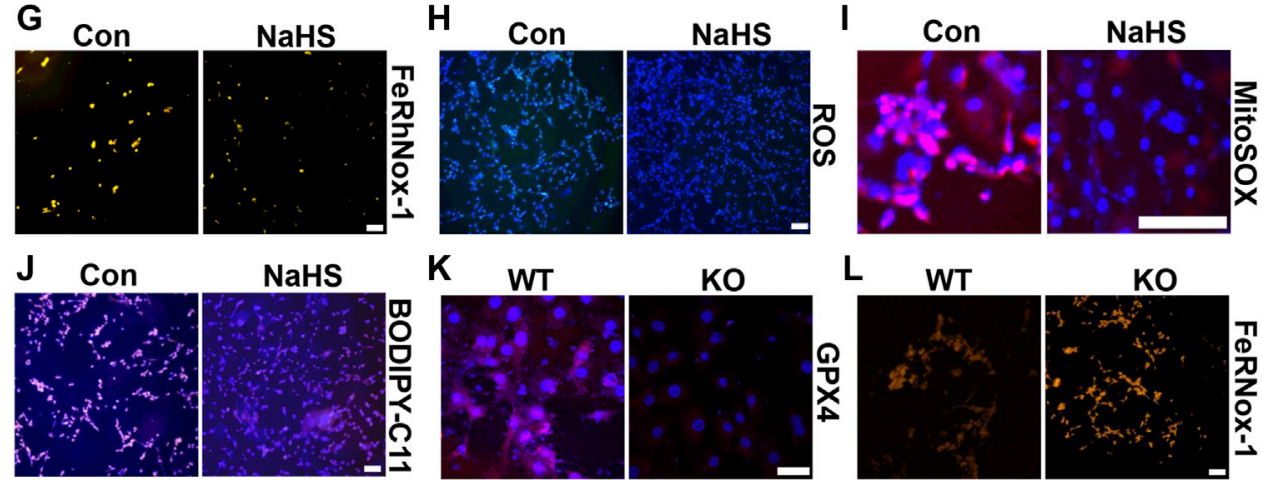

NaHS
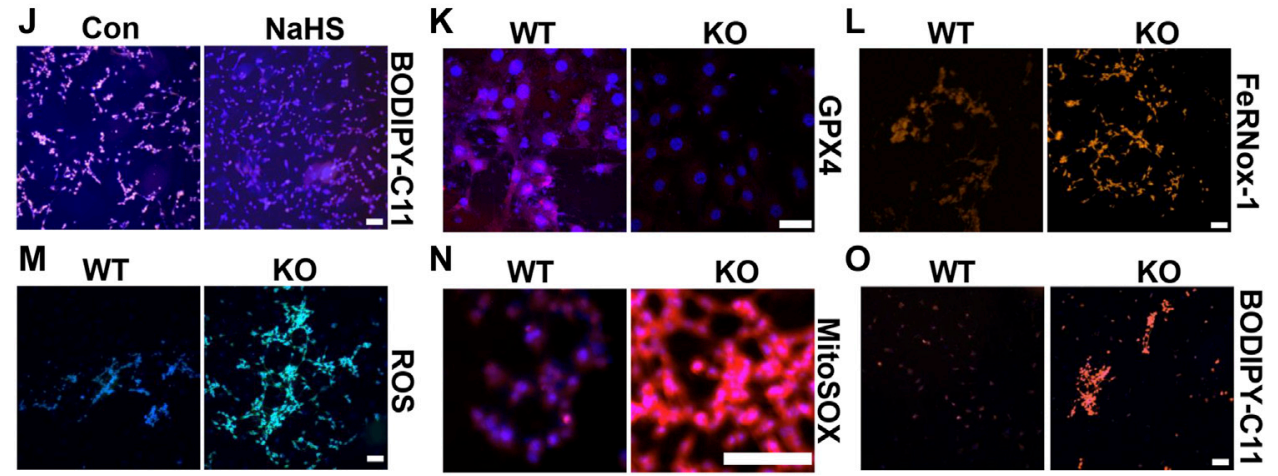

O WT

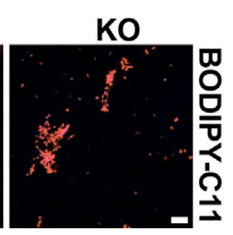

FIGURE 5 | CSE/ $\mathrm{H}_{2} \mathrm{~S}$ attenuates HHP-induced ferroptosis in VSMCs. $\mathrm{H}_{2} \mathrm{~S}$ production was measured by the modified methylene blue method under 100 or $200 \mathrm{mmHg}$ (A). Western blot assay analysis of the CSE expression under hydrostatic pressure; the left panel is the representative image, and the right panel is the statistical graph (B). CSE mRNA level under hydrostatic pressure (C). GSH assay kit measured the GSH level in response to hydrostatic pressure (D). After NaHS administration $(0.1 \mathrm{mM}, 24 \mathrm{~h})$ in the presence of $200 \mathrm{mmHg}$, the GSH content was measured (E). Representative image of GPX4 staining after NaHS treatment under HHP conditions (F). Effect of $\mathrm{NaHS}_{\text {on }} \mathrm{Fe}^{2+} \mathbf{( G )}$, ROS (H), mitochondrial ROS (I), and lipid oxidation (J) under $200 \mathrm{mmHg}$ by immunofluorescent staining. In isolated primary mouse VSMCs, the effect of deletion of CSE on the GPX4 expression (K), iron level (L), ROS production (M), mitochondrial ROS production (N), and lipid peroxidation (O) under HHP conditions by immunofluorescent staining. All scale bar $=50 \mu \mathrm{M}{ }^{\star} p<0.05,{ }^{\star \star} p<0.01,{ }^{\star \star \star} p<0.001$.

lipid oxidation (Figure 6E and Supplementary Figure S4E). In summary, our result indicated that $\mathrm{CSE} / \mathrm{H}_{2} \mathrm{~S}$ contributes to the regulation of ferroptosis under HHP conditions.

\section{DISCUSSION}

Mechanical forces play an important role in vasculature and circulation, such as rapid regulation of vascular wall elasticity, administration of vascular remodeling, and the modulation of VSMC and endothelial function (Qiu et al., 2014). Our previous study identifies a novel cellular taxonomy of VSMCs under hydrostatic pressure by single-cell RNA sequencing. HHP derived VSMCs into inflammatory and endothelial-function inhibitory VSMCs, resulting in VSMC dysfunction (cytokine secretion and angiogenesis inhibition) (Chen Z. et al., 2021). However, the underlying mechanism is unclear. In the current study, we first demonstrated that HHP induced VSMC ferroptosis evidence as iron accumulation, ROS generation, and lipid peroxidation. Furthermore, inhibiting ferroptosis by Fer-1 alleviated, promoting ferroptosis by RLS3 increased inflammatory and endothelial function inhibitory VSMC phenotypes. For the mechanism, we demonstrated that HHP downregulated $\mathrm{CSE} / \mathrm{H}_{2} \mathrm{~S}$ and then lowered GSH generation, resulting in ferroptosis. Our findings highlight that ferroptosis is a novel pathophysiological regulatory mechanism for VSMC function and as a new druggable target for therapeutic hypertension concomitant vascular diseases. 

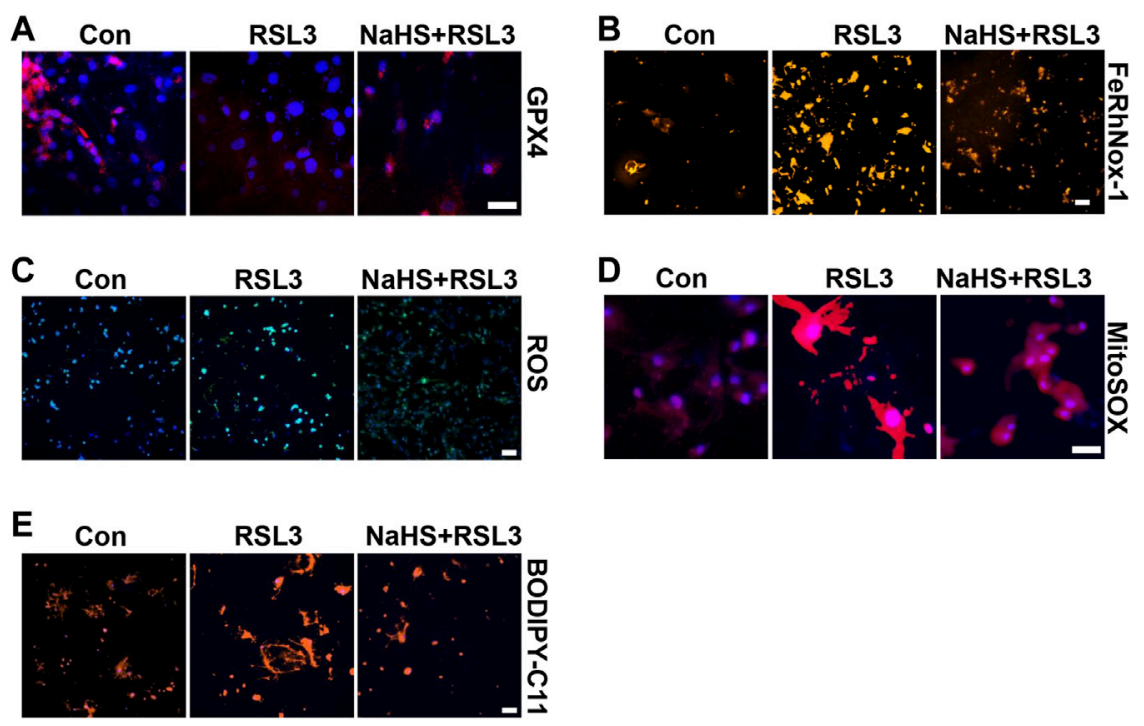

FIGURE 6 | CSE/ $\mathrm{H}_{2}$ S rescue RLS3-induced ferroptosis under HHP conditions. Under 200 mmHg conditions, HASMCs were pretreated with NaHS 24 h and followed by RLS3 treatment for another $6 \mathrm{~h}$. Immunofluorescent staining of GPX4 (A). HASMCs were incubated with the FeRhNOX-1 probe for measuring iron (B) HASMCs were labeled with the ROS probe to detect cellular ROS (C), MitoSOX Red to detect mitochondrial ROS (D), and BODIPY-C11 to detect lipid peroxidation (E) by immunofluorescent staining. All scale bar $=50 \mu \mathrm{M}$.

Hypertension is tightly associated with oxidation stress (McMaster et al., 2015; Touyz et al., 2018). An imbalance between the oxidant and antioxidant system leads to ROS production exaggeration in the arterial media from hypertension patients with hypertension and experimental animal models of hypertension (Touyz and Schiffrin, 2001). Overproduced ROS promoted VSMC proliferation, induced VSMC migration, and switched to the differentiated phenotype (Badran et al., 2020). Dedifferentiated VSMCs synthesize and secrete a repertoire of growth factors and inflammatory cytokines which is a key initiating factor for vascular remodeling. ROS promotes MMP2, MMP9, and collagen expressions and disturbs the extracellular matrix (Rajagopalan et al., 1996; Branchetti et al., 2013). Additionally, oxidation stress induces calcification, stiffness, and aging leading to VSMC dysfunction (Takaishi et al., 2021). ROS overproduction, a center of oxidation stress, is a main characteristic of ferroptosis. Our study first identified GPX4, a key enzyme of ferroptosis, which was dramatically downregulated in arterial media of SHR. In vitro, VSMCs exhibited intracellular and mitochondrial ROS overproduction, lipid peroxidation, and iron accumulation under high hydrostatic stress. Ferroptosis upregulation under high hydrostatic stress is a novel pathophysiological process in VSMC dysfunction.

Compelling evidence has indicated that ferroptosis is an essential regulator of inflammation. GPX4 deficiency blocked T-cell survival and B-cell development (Matsushita et al., 2015; Muri et al., 2019). The ferroptosis inducer erastin promoted human peripheral blood mononuclear cells differentiating into B cells and natural killer cells (Tang et al., 2021). Ferroptotic death cells recruited macrophages by secreting CCL2 and CCL7 (Wang et al., 2021a). Ferroptosis inhibitors exhibited the antiinflammation effect by suppressing TNF- $\alpha$, IL-6, and IL- $1 \beta$ releasing (Tsurusaki et al., 2019). Consistent with the previous study, Fer-1 treatment attenuated, while RLS3 treatment augmented the HHP-stimulated CXCL2 (one marker gene of inflammatory VSMCs) protein level. In addition, high hydrostatic pressure also induced $A K R 1 C 2$ (one marker gene of endothelial function inhibitory VSMCs) which was lowered by Fer-1 but enhanced by RLS3. Studies indicated ferroptosis led to endothelial dysfunction which is a critical pathogenetic factor of hypertension. Iron accumulation resulted in endothelial damage (Vinchi et al., 2020). Fer-1 administration attenuated ox-LDL-induced inflammation and endotheliocyte death, while erastin induced ROS production and ferroptotic death of HUVECs (Xiao et al., 2019; Bai et al., 2020). Combined with our results, ferroptosis may be a potential therapeutic target to hypertension via modulating endothelial function.

One important characteristic of ferroptosis is GSH depletion. $\mathrm{GSH}$, an intracellular antioxidant, is the synthesis from the homocysteine/methionine cycle (Rodrigues and Percival, 2019). GSH precursor L-cysteine is also a main source of hydrogen sulfide. Growing evidence demonstrated that $\mathrm{H}_{2} \mathrm{~S}$ enhances $\mathrm{GSH}$ production to attenuate oxidative stress. In a neurocyte, mitochondrial $\mathrm{H}_{2} \mathrm{~S}$ production increases the $\mathrm{GSH}$ level and promotes its redistribution to the mitochondria to protect the neurocyte from oxidative stress (Kimura et al., 2010). In a myotube, $\mathrm{H}_{2} \mathrm{~S}$ promotes $\mathrm{GSH}$ synthesis to improve impaired glucose homeostasis (Parsanathan and Jain, 2018). $\mathrm{H}_{2} \mathrm{~S}$ donor NaHS administration enhances GSH production to decrease oxidative stress and delay cell senescence (Yang et al., 2013). Consistently, our study showed that exogenous (NaHS administration) $\mathrm{H}_{2} \mathrm{~S}$ production significantly rescued GSH reduction in response to $\mathrm{HHP}$. Thus, $\mathrm{H}_{2} \mathrm{~S}$ downregulation-mediated $\mathrm{GSH}$ reduction may be a novel mechanism of ferroptosis under HHP. 
Recently, studies showed the protective role of $\mathrm{H}_{2} \mathrm{~S}$ is associated with ferroptosis inhibition. Wang and Chen et al. identified $\mathrm{H}_{2} \mathrm{~S}$ restrains ferroptosis via inhibiting ALOX12 acetylation and regulating the $\mathrm{xCT}$ (the functional submit of the $\mathrm{Xc}^{-}$system) stability (Chen S. et al., 2021; Wang et al., 2021c). $\mathrm{H}_{2} \mathrm{~S}$ donor GYY4137 treatment alleviates ferroptosis to attenuate acute lung injury (Li et al., 2021). Inhibition of $\mathrm{H}_{2} \mathrm{~S}$ production via the $\mathrm{CBS}$ inhibitor $\mathrm{CH} 004$ supplement aggravates ferroptosis in hepatocellular carcinoma (Wang et al., 2018). These studies suggested $\mathrm{H}_{2} \mathrm{~S}$ could restrain ferroptosis to exert a protective effect. However, experimental evidence in the cardiovascular system is still missing. Our present study illustrated that $\mathrm{H}_{2} \mathrm{~S}$ donor NaHS administration significantly increased the GPX4 expression, downregulated ROS production, and lipid peroxidation, thus reversing high hydrostatic-induced ferroptosis. In addition, NaHS rescued RLS3-induced ferroptosis. Overall, $\mathrm{H}_{2} \mathrm{~S}$ inhibits ferroptosis to attenuate HHPinduced VSMC dysfunction.

Taken together, the present study highlights the essential regulator role of ferroptosis in the HHP-triggered VSMC novel phenotype switch. The novel pathophysiological process also accounts for HHP, a direct biomechanical force, triggers VSMC ferroptosis, then drives vascular inflammation and limitation vascular relaxation, and contributes to vascular remodeling and aging even if calcified, thus exacerbating concomitant vascular damages and diseases (such as atherosclerosis). More interestingly, our study also indicates a new pathway about the ROS-GSH-iron-ferroptosis signal cascade in $\mathrm{CSE} / \mathrm{H}_{2} \mathrm{~S}$ cardiovascular protection.

\section{DATA AVAILABILITY STATEMENT}

The original contributions presented in the study are included in the article/Supplementary Material; further inquiries can be directed to the corresponding authors.

\section{REFERENCES}

Badgley, M. A., Kremer, D. M., Maurer, H. C., DelGiorno, K. E., Lee, H.-J., Purohit, V., et al. (2020). Cysteine Depletion Induces Pancreatic Tumor Ferroptosis in Mice. Science 368 (6486), 85-89. doi:10.1126/ science.aaw9872

Badran, A., Nasser, S. A., Mesmar, J., El-Yazbi, A. F., Bitto, A., Fardoun, M. M., et al. (2020). Reactive Oxygen Species: Modulators of Phenotypic Switch of Vascular Smooth Muscle Cells. Int. J. Mol. Sci. 21 (22), 8764. doi:10.3390/ ijms21228764

Bai, T., Li, M., Liu, Y., Qiao, Z., and Wang, Z. (2020). Inhibition of Ferroptosis Alleviates Atherosclerosis through Attenuating Lipid Peroxidation and Endothelial Dysfunction in Mouse Aortic Endothelial Cell. Free Radic. Biol. Med. 160, 92-102. doi:10.1016/j.freeradbiomed.2020.07.026

Branchetti, E., Poggio, P., Sainger, R., Shang, E., Grau, J. B., Jackson, B. M., et al. (2013). Oxidative Stress Modulates Vascular Smooth Muscle Cell Phenotype via CTGF in Thoracic Aortic Aneurysm. Cardiovasc. Res. 100 (2), 316-324. doi:10. $1093 /$ cvr/cvt205

Chen, S., Bu, D., Zhu, J., Yue, T., Guo, S., Wang, X., et al. (2021). Endogenous Hydrogen Sulfide Regulates xCT Stability through Persulfidation of OTUB1 at Cysteine 91 in colon Cancer Cells. Neoplasia 23 (5), 461-472. doi:10.1016/j.neo. 2021.03.009

\section{ETHICS STATEMENT}

The studies involving human participants were reviewed and approved by the Institutional Animal Care and Use Committee, the Experimental Animal Center, Fuwai Hospital, National Center for Cardiovascular Diseases, China. The ethics committee waived the requirement of written informed consent for participation. The animal study was reviewed and approved by the Institutional Animal Care and Use Committee, the Experimental Animal Center, Fuwai Hospital, National Center for Cardiovascular Diseases, China.

\section{AUTHOR CONTRIBUTIONS}

$\mathrm{ZC}$ and $\mathrm{BG}$ conceived the project and designed the experiments. $\mathrm{RJ}$ conducted most of the experiments and data analyses. RY conducted a part of experiments. $\mathrm{CC}$ and $\mathrm{HZ}$ provided the technical assistance. The manuscript was written by $\mathrm{ZC}$ and revised and edited by BG and JC.

\section{FUNDING}

The study was supported by the CAMS Innovation Fund for Medical Sciences (CIFMS, 2021-I2M-1-007), the National Natural Science Foundation of China $(81800367,81870318,81825002)$, the National Key R\&D Program of China (2018YFC1312703), and the Beijing Outstanding Young Scientist Program (BJJWZYJH01201910023029).

\section{SUPPLEMENTARY MATERIAL}

The Supplementary Material for this article can be found online at: https://www.frontiersin.org/articles/10.3389/fcell.2022.829316/ full\#supplementary-material

Chen, Z., Zhang, H., Bai, Y., Cui, C., Li, S., Wang, W., et al. (2021). Single Cell Transcriptomic Analysis Identifies Novel Vascular Smooth Muscle Subsets under High Hydrostatic Pressure. Sci. China Life Sci. 64 (10), 1677-1690. doi:10.1007/s11427-020-1852-x

Cheng, W.-P., Wang, B.-W., Chen, S.-C., Chang, H., and Shyu, K.-G. (2012). Mechanical Stretch Induces the Apoptosis Regulator PUMA in Vascular Smooth Muscle Cells. Cardiovasc. Res. 93 (1), 181-189. doi:10.1093/cvr/cvr280

Dixon, S. J., Lemberg, K. M., Lamprecht, M. R., Skouta, R., Zaitsev, E. M., Gleason, C. E., et al. (2012). Ferroptosis: an Iron-dependent Form of Nonapoptotic Cell Death. Cell 149 (5), 1060-1072. doi:10.1016/j.cell.2012.03.042

Gujja, P., Rosing, D. R., Tripodi, D. J., and Shizukuda, Y. (2010). Iron Overload Cardiomyopathy. J. Am. Coll. Cardiol. 56 (13), 1001-1012. doi:10.1016/j.jacc. 2010.03.083

Hayashi, K., and Naiki, T. (2009). Adaptation and Remodeling of Vascular wall; Biomechanical Response to Hypertension. J. Mech. Behav. Biomed. Mater. 2 (1), 3-19. doi:10.1016/j.jmbbm.2008.05.002

Kimura, H. (2021). Hydrogen Sulfide (H2S) and Polysulfide (H2Sn) Signaling: The First 25 Years. Biomolecules 11 (6), 896. doi:10.3390/biom11060896

Kimura, Y., Goto, Y.-I., and Kimura, H. (2010). Hydrogen Sulfide Increases Glutathione Production and Suppresses Oxidative Stress in Mitochondria. Antioxid. Redox Signaling 12 (1), 1-13. doi:10.1089/ars.2008.2282

Li, J., Li, M., Li, L., Ma, J., Yao, C., and Yao, S. (2022). Hydrogen Sulfide Attenuates Ferroptosis and Stimulates Autophagy by Blocking mTOR Signaling in Sepsis- 
Induced Acute Lung Injury. Mol. Immunol. 141, 318-327. doi:10.1016/j. molimm.2021.12.003

Li, N., Wang, W., Zhou, H., Wu, Q., Duan, M., Liu, C., et al. (2020). Ferritinophagymediated Ferroptosis Is Involved in Sepsis-Induced Cardiac Injury. Free Radic. Biol. Med. 160, 303-318. doi:10.1016/j.freeradbiomed.2020.08.009

Liu, C., Li, B., Yan, Q., Niu, S., Zhao, Y., Xiong, C., et al. (2021). Protective Effects and Mechanisms of Recombinant Human Glutathione Peroxidase 4 on Isoproterenol-Induced Myocardial Ischemia Injury. Oxidative Med. Cell Longevity 2021, 1-17. doi:10.1155/2021/6632813

Matsushita, M., Freigang, S., Schneider, C., Conrad, M., Bornkamm, G. W., and Kopf, M. (2015). T Cell Lipid Peroxidation Induces Ferroptosis and Prevents Immunity to Infection. J. Exp. Med. 212 (4), 555-568. doi:10.1084/jem.20140857

McMaster, W. G., Kirabo, A., Madhur, M. S., and Harrison, D. G. (2015). Inflammation, Immunity, and Hypertensive End-Organ Damage. Circ. Res. 116 (6), 1022-1033. doi:10.1161/CIRCRESAHA.116.303697

Muri, J., Thut, H., Bornkamm, G. W., and Kopf, M. (2019). B1 and Marginal Zone B Cells but Not Follicular B2 Cells Require Gpx4 to Prevent Lipid Peroxidation and Ferroptosis. Cel Rep. 29 (9), 2731-2744.e4. doi:10.1016/j.celrep.2019.10.070

Niu, Y., Du, C., Cui, C., Zhang, H., Deng, Y., Cai, J., et al. (2021). Norswertianolin Promotes Cystathionine $\gamma$-Lyase Activity and Attenuates Renal Ischemia/ Reperfusion Injury and Hypertension. Front. Pharmacol. 12, 677212. doi:10. 3389/fphar.2021.677212

Parsanathan, R., and Jain, S. K. (2018). Hydrogen Sulfide Increases Glutathione Biosynthesis, and Glucose Uptake and Utilisation in C2C12 Mouse Myotubes. Free Radic. Res. 52 (2), 288-303. doi:10.1080/10715762.2018.1431626

Qi, Y.-X., Han, Y., and Jiang, Z.-L. (2018). Mechanobiology and Vascular Remodeling: From Membrane to Nucleus. Adv. Exp. Med. Biol. 1097, 69-82. doi:10.1007/978-3-319-96445-4_4

Qiu, J., Zheng, Y., Hu, J., Liao, D., Gregersen, H., Deng, X., et al. (2014). Biomechanical Regulation of Vascular Smooth Muscle Cell Functions: from In Vitro to In Vivo Understanding. J. R. Soc. Interf. 11 (90), 20130852. doi:10. 1098/rsif.2013.0852

Qiu, Y., Cao, Y., Cao, W., Jia, Y., and Lu, N. (2020). The Application of Ferroptosis in Diseases. Pharmacol. Res. 159, 104919. doi:10.1016/j.phrs.2020.104919

Rajagopalan, S., Meng, X. P., Ramasamy, S., Harrison, D. G., and Galis, Z. S. (1996). Reactive Oxygen Species Produced by Macrophage-Derived Foam Cells Regulate the Activity of Vascular Matrix Metalloproteinases In Vitro. Implications for Atherosclerotic Plaque Stability. J. Clin. Invest. 98 (11), 2572-2579. doi:10.1172/JCI119076

Rodrigues, C., and Percival, S. (2019). Immunomodulatory Effects of Glutathione, Garlic Derivatives, and Hydrogen Sulfide. Nutrients 11 (2), 295. doi:10.3390/ nu11020295

Stockwell, B. R., Friedmann Angeli, J. P., Bayir, H., Bush, A. I., Conrad, M., Dixon, S. J., et al. (2017). Ferroptosis: A Regulated Cell Death Nexus Linking Metabolism, Redox Biology, and Disease. Cell 171 (2), 273-285. doi:10.1016/j.cell.2017.09.021

Tabassum, R., and Jeong, N. Y. (2019). Potential for Therapeutic Use of Hydrogen Sulfide in Oxidative Stress-Induced Neurodegenerative Diseases. Int. J. Med. Sci. 16 (10), 1386-1396. doi:10.7150/ijms.36516

Takaishi, K., Kinoshita, H., Kawashima, S., and Kawahito, S. (2021). Human Vascular Smooth Muscle Function and Oxidative Stress Induced by NADPH Oxidase with the Clinical Implications. Cells 10 (8), 1947. doi:10.3390/ cells10081947

Takayama, K., Ogawa, M., Mochizuki, M., and Takeuchi, M. (2013). Cytomegalovirus Retinitis in a Patient with Proliferative Diabetes Retinopathy. Ocul. Immunol. Inflamm. 21 (3), 225-226. doi:10.3109/09273948.2012.762983

Tang, D., Chen, X., Kang, R., and Kroemer, G. (2021). Ferroptosis: Molecular Mechanisms and Health Implications. Cell Res 31 (2), 107-125. doi:10.1038/ s41422-020-00441-1

Touyz, R. M., Alves-Lopes, R., Rios, F. J., Camargo, L. L., Anagnostopoulou, A., Arner, A., et al. (2018). Vascular Smooth Muscle Contraction in Hypertension. Cardiovasc. Res. 114 (4), 529-539. doi:10.1093/cvr/cvy023
Touyz, R. M., and Schiffrin, E. L. (2001). Increased Generation of Superoxide by Angiotensin II in Smooth Muscle Cells from Resistance Arteries of Hypertensive Patients: Role of Phospholipase D-dependent NAD(P)H Oxidase-Sensitive Pathways. J. Hypertens. 19 (7), 1245-1254. doi:10.1097/ 00004872-200107000-00009

Tsurusaki, S., Tsuchiya, Y., Koumura, T., Nakasone, M., Sakamoto, T., Matsuoka, M., et al. (2019). Hepatic Ferroptosis Plays an Important Role as the Trigger for Initiating Inflammation in Nonalcoholic Steatohepatitis. Cell Death Dis 10 (6), 449. doi:10.1038/s41419-019-1678-y

Vinchi, F., Porto, G., Simmelbauer, A., Altamura, S., Passos, S. T., Garbowski, M., et al. (2020). Atherosclerosis Is Aggravated by Iron Overload and Ameliorated by Dietary and Pharmacological Iron Restriction. Eur. Heart J. 41 (28), 2681-2695. doi:10.1093/eurheartj/ehz112

Wang, L., Cai, H., Hu, Y., Liu, F., Huang, S., Zhou, Y., et al. (2018). A Pharmacological Probe Identifies Cystathionine $\beta$-synthase as a New Negative Regulator for Ferroptosis. Cel Death Dis 9 (10), 1005. doi:10.1038/ s41419-018-1063-2

Wang, Y., Quan, F., Cao, Q., Lin, Y., Yue, C., Bi, R., et al. (2021a). Quercetin Alleviates Acute Kidney Injury by Inhibiting Ferroptosis. J. Adv. Res. 28, 231-243. doi:10.1016/j.jare.2020.07.007

Wang, Y., Wang, S., Xin, Y., Zhang, J., Wang, S., Yang, Z., et al. (2021b). Hydrogen Sulfide Alleviates the Anxiety-like and Depressive-like Behaviors of Type 1 Diabetic Mice via Inhibiting Inflammation and Ferroptosis. Life Sci. 278, 119551. doi:10.1016/j.lfs.2021.119551

Wang, Y., Yu, R., Wu, L., and Yang, G. (2021c). Hydrogen Sulfide Guards Myoblasts from Ferroptosis by Inhibiting ALOX12 Acetylation. Cell Signal. 78, 109870. doi:10.1016/j.cellsig.2020.109870

Wu, X., Li, Y., Zhang, S., and Zhou, X. (2021). Ferroptosis as a Novel Therapeutic Target for Cardiovascular Disease. Theranostics 11 (7), 3052-3059. doi:10.7150/ thno.54113

Xiao, F.-J., Zhang, D., Wu, Y., Jia, Q.-H., Zhang, L., Li, Y.-X., et al. (2019). miRNA17-92 Protects Endothelial Cells from Erastin-Induced Ferroptosis through Targeting the A20-ACSL4 axis. Biochem. Biophysical Res. Commun. 515 (3), 448-454. doi:10.1016/j.bbrc.2019.05.147

Yang, G., Zhao, K., Ju, Y., Mani, S., Cao, Q., Puukila, S., et al. (2013). Hydrogen Sulfide Protects against Cellular Senescence via S-Sulfhydration of Keap1 and Activation of Nrf2. Antioxid. Redox Signaling 18 (15), 1906-1919. doi:10.1089/ ars.2012.4645

Yang, W. S., and Stockwell, B. R. (2016). Ferroptosis: Death by Lipid Peroxidation. Trends Cel Biol. 26 (3), 165-176. doi:10.1016/j.tcb.2015.10.014

Zhou, J., Li, Y.-S., and Chien, S. (2014). Shear Stress-Initiated Signaling and its Regulation of Endothelial Function. Arterioscler Thromb. Vasc. Biol. 34 (10), 2191-2198. doi:10.1161/ATVBAHA.114.303422

Conflict of Interest: The authors declare that the research was conducted in the absence of any commercial or financial relationships that could be construed as a potential conflict of interest.

Publisher's Note: All claims expressed in this article are solely those of the authors and do not necessarily represent those of their affiliated organizations, or those of the publisher, the editors, and the reviewers. Any product that may be evaluated in this article, or claim that may be made by its manufacturer, is not guaranteed or endorsed by the publisher.

Copyright $(2022$ Jin, Yang, Cui, Zhang, Cai, Geng and Chen. This is an open-access article distributed under the terms of the Creative Commons Attribution License (CC $B Y)$. The use, distribution or reproduction in other forums is permitted, provided the original author(s) and the copyright owner(s) are credited and that the original publication in this journal is cited, in accordance with accepted academic practice. No use, distribution or reproduction is permitted which does not comply with these terms. 